\title{
Bone Marrow Very Small Embryonic-Like Stem Cells: New Generation of Autologous Cell Therapy Soon Ready for Prime Time?
}

\author{
David M. Smadja ${ }^{1,2,3}$
}

Published online: 18 January 2017

(C) Springer Science+Business Media New York 2017

\begin{abstract}
Very small embryonic-like stem cells (VSELs) are major pluripotent stem cells described in human and mouse. In this issue of Stem Cell Reviews and Reports, Shaikh and colleagues show in a valuable work that mouse bone marrow collected after $5 \mathrm{FU}$ treatment contains VSELs able to undergo in vitro multi-lineage differentiation into cells from all three germ layers and also in germ and hematopoietic cells. These findings are robust since no confounding factor such as feeder cell fusion with VSELs can occur here. This paper allows one to better appreciate bone marrow-VSELs differentiation potential and opens new perspectives for autologous cell therapy. Furthermore, it might help explaining lots of contradictive data from the past 20 years, in particular related to ability of bone marrow cells to differentiate into cardiomyocytes.
\end{abstract}

\section{Editorial}

Very small embryonic-like stem cells (VSELs) are major pluripotent stem cells defined in humans as Lineage-negative, CD133positive, and CD45-negative cells of small size $(<6 \mathrm{~mm}$ in diameter) [1]. VSELs have been shown in several studies to be able to differentiate into cells from different germ layers. VSELs have a large nucleus-to-cytoplasm ratio, and a high expression level of pluripotency core factors. They could represent a clinically relevant alternative to embryonic stem cells (ESCs) and induced

David M. Smadja

david.smadja@aphp.fr

1 Hematology Department, AP-HP, European Georges Pompidou Hospital, 20 rue Leblanc, 75015 Paris, France

2 Université Paris Descartes, Sorbonne Paris Cité, Paris, France

3 Inserm UMR-S1140, Paris, France pluripotent stem cells (iPS) for cell therapy since VSELs do not complete blastocyst development and do not form teratomas after transplantation into deficient mice $[2,3]$.

In this issue of Stem Cell Reviews and Reports, Shaikh and colleagues [4] provide both potential mechanistic insights and clinically relevant informations about VSELs differentiation. Indeed, they demonstrate for the first time in a single study that mouse bone marrow, after a $5 \mathrm{FU}$ treatment, was enriched in VSELs that underwent multi-lineage differentiation. Indeed, VSELs differentiated into cells from all three germ layers in vitro but also in germ and hematopoietic cells, thereby fulfilling the criteria for pluripotency as illustrated in Figure 1. When GFP-positive VSELs were co-cultured with non-GFP feeder support (or vice versa) in cardiac, neuronal- or pancreatic differentiation media but also with sertoli-conditioned media containing Follicle-Stimulating Hormone, GFP-positive cells were shown to differentiate into cardiomyocytes, neuronal, pancreatic and germ cells, respectively. Moreover, Shaikh et al. demonstrated that 5-FU enriched VSELs were also able to give rise to hematopoietic cells after a differentiation step on OP-9 cells, thus confirming the results from Ratajczak's group [5]. This pluripotent feature of 5-FU enriched VSELs provides further evidence that VSELs are the most primitive stem cells in bone marrow which can give rise to different tissue committed progenitor cells from all germ layers without confounding factor of feeder cell fusion with VSELs. This study does not only demonstrate that the bone marrow-VSEL source obtained after 5-FU treatment can undergo hematopoietic and nonhematopoietic differentiation supporting the evidence of their pluripotent state, but also opens a new area of therapeutic perspective. Finally, it helps explaining lots of confusing data from the literature of the past 20 years.

First, the pluripotent state of bone marrow derived-VSELs might have some consequences on bone marrow transplantation and its indications and/or therapeutic applications. VSELs are 
Fig. 1 Migratory primordial germ cells (PGCs) could be at the origin of very small embryonic like cells (VSELs). VSELs are present in adult tissues, including bone marrow, and are able to undergo in vitro multi-lineage differentiation into cells from all three germ layers and also in germ and hematopoietic cells. $\rightarrow$ Differentiation process described by Shaikh and colleagues or in other publications to date. - - - > Differentiation process yet to be determined

\section{Primordial germ cells (PGCs)} Migration in bone marrow (and tissues)

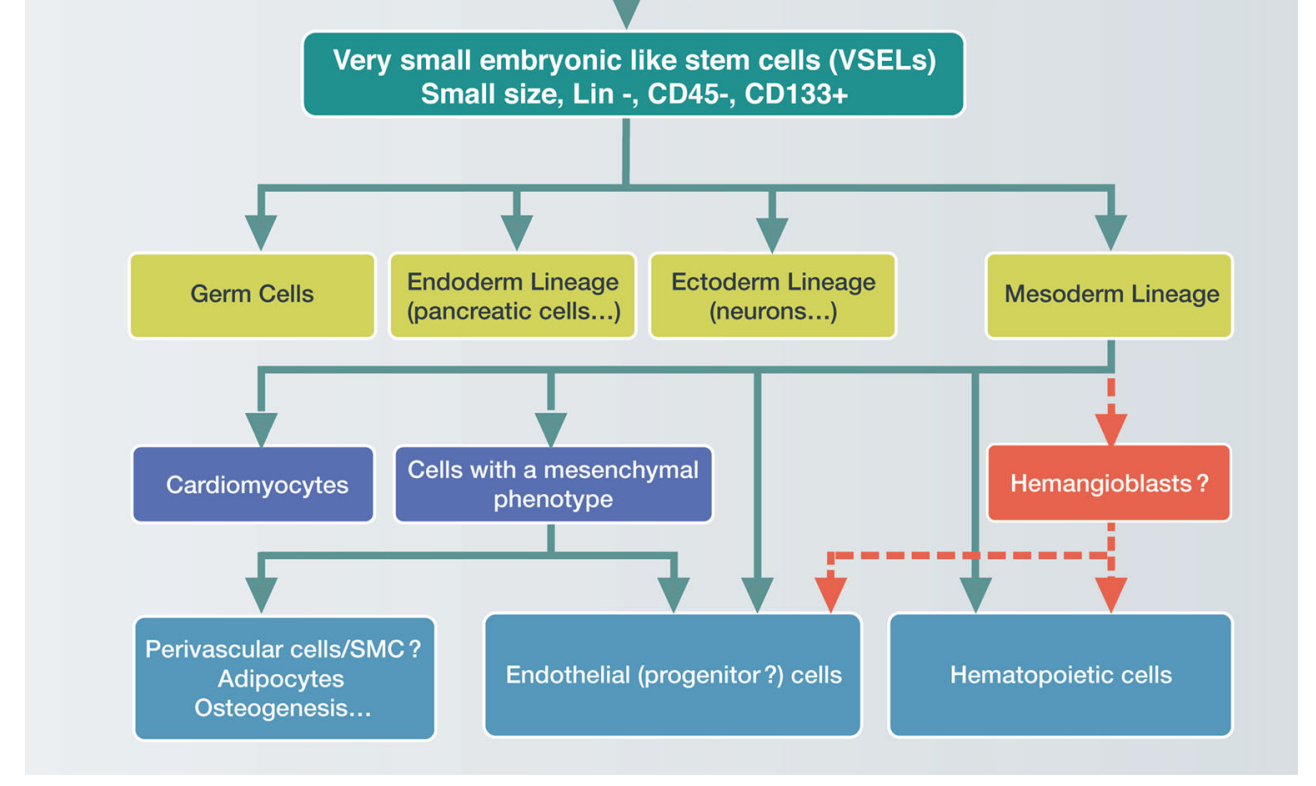

unknowingly discarded during processing of autologous or allogenic bone marrow and/or cord blood transplantation because of their very small size and high nucleus-to-cytoplasm ratio. VSELs enrichment of bone marrow transplants would be likely to provide a better engraftment after transplantation. Furthermore, since they have been described to be mobilized in peripheral blood in several situations [6-8], VSELs could help to cure damages of transplantation preconditioning, visceral failure (example of BM transplantation in Systemic sclerosis) and to prevent organ failure because of their tissue regeneration ability. This hypothesis of VSELs involvement in extra-hematopoietic recovery after bone marrow transplantation worths to be explored in the next years. Since VSELs have also been described in mobilized peripheral blood [6] that has become the predominant stem cell source for allogeneic and autologous hematopoietic cell transplantation, VSELs count in graft could also be tested as prognostic factor for long-term survival after peripheral blood hematopoietic stem cell transplantation. This study also emphasizes that VSELs isolated from bone marrow, and not only from gonads, can spontaneously differentiate into germ cells, providing explanation for earlier publications reporting bone marrow as a source of germ cells $[9,10]$. This ability to give rise to germ cells could also be used in VSELs-enriched BM graft since the occurrence of late sequelae after myeloablative conditioning regimens for stem-cell transplantation has been shown to induce germinal toxicity and spare fertility.

Secondly, BM-VSELs pluripotent capacity could allow resume the debate about BM cell therapy in some organs and contradictive data described in the past 20 years. Indeed, it has become evident that hematopoietic stem cells lack plasticity and transdifferentiation potential. One example is bone marrow-derived or cardiac-derived stem cell therapy for heart disease. While it has demonstrated safety and efficacy in clinical trials, functional improvements have been shown limited. One of the first papers demonstrating that bone marrow cells injected into the heart could differentiate into cardiomyocytes and improve cardiac function has been published in Nature in 2011 [11]. After this first paper, several groups [12-14] showed that HSCs do not transdifferentiate into cardiomyocytes. However, clinical trials have been initiated (recently metaanalyzed in [15]) while this experimental conclusion was not confirmed by others. The studies have been carried on despite inconsistent results and when positive, the effects observed were usually transient and probably a consequence of paracrine effects more than of a true cellular incorporation. Thus, one hypothesis is that VSELs could explain discrepancies in studies regarding cardiomyocyte differentiation from BM. If HSC do not have cardiomyocyte (CM) differentiation ability, VSELs contained in whole BM or in peripheral mobilized blood could give rise to $\mathrm{CM}$ and explain part of Orlic et al. data obtained in 2001. Moreover, cell isolation protocols have been already demonstrated to have a major impact on the functional activity of BM-derived progenitor cells [16]. Since Ratajczak's group also described different VSELs composition depending on the cell preparation [17], additional functional testing, and in particular VSELs composition, could be mandatory to analyze and go forward clinical cell therapy trials.

While there is clear evidence that HSC do not give rise to $\mathrm{CM}$, and because before VSELs description [18] no effective autologous CM population could be obtained, allogenic 
human embryonic stem cell (hESC)-derived cardiac progenitors are currently used in a clinical trial directed by $\operatorname{Pr}$ Menasche in patients suffering from severe ischemic Left Ventricular dysfunction [19, 20]. There is compelling evidence that cells committed to a cardiac lineage are most effective for improving the function of infarcted hearts than HSC or myoblasts. Expansion of pluripotent hESC clone under good manufacturing practice (GMP) conditions has been done and after a growth factor-induced cardiac specification and a purification step of committed cells by immunomagnetic sorting, stage-specific embryonic antigen (SSEA)-1-positive cell population have been incorporated into a fibrin scaffold. This patch delivers cells after a surgical epicardial implantation, and is more effective than hand-held multiple intramyocardial injections [21]. This allogenic cell delivery in patients requires immunosuppressive treatment. If human bone marrowVSELs can give rise to CM under GMP conditions, the same clinical trial could be done using autologous VSELs derived$\mathrm{CM}$ without the inconvenient of immunosuppressive therapy in elderly people with cardiac disease. Human ESC-derived differentiated cells have already been used in patients with spinal cord injury [22] and ocular diseases [23] and VSELs could also be an autologous cell therapy approach in these disease, re-opening area of autologous stem cell therapy.

VSELs ability to differentiate into several lineages can also explain the discrepancies observed in the literature in the field of vascular regeneration using endothelial progenitor cells (EPCs). Indeed, vasculogenic EPCs (also named late-EPC or ECFCs for endothelial colony forming cells) have been described in an elegant study of BM transplantation from Hebbel's group [24]. However, Yoder's group in Indianapolis proposed that these ECFCs directly come from vessels [25, 26] and from CD45 negative cells [27-29]. VSELs have a huge motility and are theoretically able to migrate from bone marrow to vessels. They have all the characteristics of potential vasculogenic CD45negative stem cells able to give rise to vessels since they have been described to differentiate in endothelial cells by several independent groups with human, mouse or rat VSELs [6, 30, 31].

In conclusion, Shaikh and colleagues fill a gap in our knowledge by identifying a large pluripotent differentiation ability of bone marrow murine VSELs. As a prerequisite to clinical trials, this pluripotent differentiation ability of bone marrow-VSELs needs now to be followed in human by developing a process to efficiently expand VSELs under GMP conditions. Indeed, the ability of VSEL to proliferate in vitro is very limited $[2,6]$. Hence, further work is required to stimulate the proliferative capacity of VSELs without affecting their differentiation potential. This step of proliferation and VSELs differentiation has a huge importance for regenerative medicine. The critical expansion step should be to reverse VSELs-quiescent state. As soon as VSELs proliferation and expansion ex vivo is possible, VSEL-autologous cell therapy trials will be ready for "prime time"!

Acknowledgements David M. Smadja research is supported by grants from the Conny-Maeva Charitable Foundation.

\section{Compliance with Ethical Standards}

Declaration of Interests Author declares no conflict of interest related to this work.

\section{References}

1. Ratajczak, M. Z., Zuba-Surma, E. K., Shin, D. M., Ratajczak, J., \& Kucia, M. (2008). Very small embryonic-like (VSEL) stem cells in adult organs and their potential role in rejuvenation of tissues and longevity. Experimental Gerontology, 43(11), 1009-17.

2. Kassmer, S. H., \& Krause, D. S. (2013). Very small embryonic-like cells: biology and function of these potential endogenous pluripotent stem cells in adult tissues. Molecular Reproduction \& Development, 80(8), 677-90.

3. Kucia, M., Zuba-Surma, E. K., Wysoczynski, M., Wu, W., Ratajczak, J., Machalinski, B., et al. (2007). Adult marrowderived very small embryonic-like stem cells and tissue engineering. Expert Opinion on Biological Therapy, 7(10), 1499-514.

4. Shaikh, A., Anand, S., Kapoor, S., Ganguly, R., \& Bhartiya, D. (2017). Mouse bone marrow VSELs exhibit differentiation into three embryonnic germ lineages and germ and hematopoietic cells in culture. Stem cells Reviews and Reports 23(1):In press.

5. Ratajczak, J., Wysoczynski, M., Zuba-Surma, E., Wan, W., Kucia, M., Yoder, M. C., et al. (2011). Adult murine bone marrow-derived very small embryonic-like stem cells differentiate into the hematopoietic lineage after coculture over OP9 stromal cells. Experimental Hematology, 39(2), 225-37.

6. Guerin, C. L., Loyer, X., Vilar, J., Cras, A., Mirault, T., Gaussem, P., et al. (2015). Bone-marrow-derived very small embryonic-like stem cells in patients with critical leg ischaemia: evidence of vasculogenic potential. Thrombosis \& Haemostasis, 113(5), 1084-94.

7. Wojakowski, W., Tendera, M., Kucia, M., Zuba-Surma, E., Paczkowska, E., Ciosek, J., et al. (2009). Mobilization of bone marrow-derived Oct-4+ SSEA-4+ very small embryonic-like stem cells in patients with acute myocardial infarction. Journal of the American College of Cardiology, 53(1), 1-9.

8. Zbucka-Kretowska, M., Eljaszewicz, A., Lipinska, D., Grubczak, K., Rusak, M., Mrugacz, G., et al. (2016). Effective mobilization of very small embryonic-like stem cells and hematopoietic stem/progenitor cells but not endothelial progenitor cells by follicle-stimulating hormone therapy. Stem Cells International, 2016, 8530207.

9. Johnson, J., Bagley, J., Skaznik-Wikiel, M., Lee, H. J., Adams, G. B., Niikura, Y., et al. (2005). Oocyte generation in adult mammalian ovaries by putative germ cells in bone marrow and peripheral blood. Cell, 122(2), 303-15.

10. Nayernia, K., Lee, J. H., Drusenheimer, N., Nolte, J., Wulf, G., Dressel, R., et al. (2006). Derivation of male germ cells from bone marrow stem cells. Laboratory Investigation, 86(7), 654-63.

11. Orlic, D., Kajstura, J., Chimenti, S., Jakoniuk, I., Anderson, S. M., $\mathrm{Li}, \mathrm{B}$., et al. (2001). Bone marrow cells regenerate infarcted myocardium. Nature, 410(6829), 701-5.

12. Murry, C. E., Soonpaa, M. H., Reinecke, H., Nakajima, H., Nakajima, H. O., Rubart, M., et al. (2004). Haematopoietic stem 
cells do not transdifferentiate into cardiac myocytes in myocardial infarcts. Nature, 428(6983), 664-8.

13. Balsam, L. B., Wagers, A. J., Christensen, J. L., Kofidis, T., Weissman, I. L., \& Robbins, R. C. (2004). Haematopoietic stem cells adopt mature haematopoietic fates in ischaemic myocardium. Nature, 428(6983), 668-73.

14. Nygren, J. M., Jovinge, S., Breitbach, M., Sawen, P., Roll, W., Hescheler, J., et al. (2004). Bone marrow-derived hematopoietic cells generate cardiomyocytes at a low frequency through cell fusion, but not transdifferentiation. Nature Medicine, 10(5), 494-501.

15. Fisher, S.,A., Doree, C., Mathur, A., Taggart, D. P., \& MartinRendon, E. (2016). Stem cell therapy for chronic ischaemic heart disease and congestive heart failure. Cochrane Database System Review 12:In press.

16. Seeger, F. H., Tonn, T., Krzossok, N., Zeiher, A. M., \& Dimmeler, S. (2007). Cell isolation procedures matter: a comparison of different isolation protocols of bone marrow mononuclear cells used for cell therapy in patients with acute myocardial infarction. European Heart Journal, 28(6), 766-72.

17. Zuba-Surma, E. K., \& Ratajczak, M. Z. (2010). Overview of very small embryonic-like stem cells (VSELs) and methodology of their identification and isolation by flow cytometric methods. Current Protocols Cytometry. Chapter 9:Unit9 29.

18. Wojakowski, W., Tendera, M., Kucia, M., Zuba-Surma, E., Milewski, K., Wallace-Bradley, D., et al. (2010). Cardiomyocyte differentiation of bone marrow-derived Oct-4 + CXCR4 + SSEA$1+$ very small embryonic-like stem cells. International Journal of Oncology, 37(2), 237-47.

19. Menasche, P., Vanneaux, V., Fabreguettes, J. R., Bel, A., Tosca, L., Garcia, S., et al. (2015). Towards a clinical use of human embryonic stem cell-derived cardiac progenitors: a translational experience. European Heart Journal, 36(12), 743-50.

20. Menasche, P., Vanneaux, V., Hagege, A., Bel, A., Cholley, B., Cacciapuoti, I., et al. (2015). Human embryonic stem cell-derived cardiac progenitors for severe heart failure treatment: first clinical case report. European Heart Journal, 36(30), 2011-7.

21. Hamdi, H., Furuta, A., Bellamy, V., Bel, A., Puymirat, E., Peyrard, S., et al. (2009). Cell delivery: intramyocardial injections or epicardial deposition? A head-to-head comparison. Annals of Thoracic Surgery, 87(4), 1196-203.
22. Shroff, G. (2016). Human embryonic stem cell therapy in chronic spinal cord injury: a retrospective study. Clinical and Translational Science, 9(3), 168-75.

23. Schwartz, S. D., Regillo, C. D., Lam, B. L., Eliott, D., Rosenfeld, P. J., Gregori, N. Z., et al. (2014). Human embryonic stem cell-derived retinal pigment epithelium in patients with age-related macular degeneration and Stargardt's macular dystrophy: follow-up of two open-label phase 1/2 studies. Lancet, 385(9967), 509-16.

24. Lin, Y., Weisdorf, D. J., Solovey, A., \& Hebbel, R. P. (2000). Origins of circulating endothelial cells and endothelial outgrowth from blood. Journal of Clinical Investigation, 105(1), 71-7.

25. Ingram, D. A., Mead, L. E., Moore, D. B., Woodard, W., Fenoglio, A., \& Yoder, M. C. (2005). Vessel wall-derived endothelial cells rapidly proliferate because they contain a complete hierarchy of endothelial progenitor cells. Blood, 105(7), 2783-6.

26. Yoder, M. C. (2010). Is endothelium the origin of endothelial progenitor cells? Arteriosclerosis, Thrombosis, and Vascular Biology, 30(6), 1094-103.

27. Yoder, M. C., Mead, L. E., Prater, D., Krier, T. R., Mroueh, K. N., $\mathrm{Li}, \mathrm{F}$., et al. (2007). Redefining endothelial progenitor cells via clonal analysis and hematopoietic stem/progenitor cell principals. Blood, 109(5), 1801-9.

28. Case, J., Mead, L. E., Bessler, W. K., Prater, D., White, H. A., Saadatzadeh, M. R., et al. (2007). Human CD34 + AC133 + VEGFR$2+$ cells are not endothelial progenitor cells but distinct, primitive hematopoietic progenitors. Experimental Hematology, 35(7), 1109-18.

29. Timmermans, F., Van Hauwermeiren, F., De Smedt, M., Raedt, R., Plasschaert, F., De Buyzere, M. L., et al. (2007). Endothelial outgrowth cells are not derived from CD133+ cells or CD45+ hematopoietic precursors. Arteriosclerosis, Thrombosis, and Vascular Biology, 27(7), 1572-9.

30. Havens, A. M., Sun, H., Shiozawa, Y., Jung, Y., Wang, J., Mishra, A., et al. (2014). Human and murine very small embryonic-like cells represent multipotent tissue progenitors, in vitro and in vivo. Stem Cells and Development, 23(7), 689-701.

31. Wu, J. H., Wang, H. J., Tan, Y. Z., \& Li, Z. H. (2012). Characterization of rat very small embryonic-like stem cells and cardiac repair after cell transplantation for myocardial infarction. Stem Cells and Development, 21(8), 1367-79. 